\title{
Biocompatible 3D Printed Microneedles for Transdermal, Intradermal, and Percutaneous Applications
}

\author{
Khalil Moussi, Abdullah Bukhamsin, Tania Hidalgo, and Jurgen Kosel:-
}

Microneedles (MNs) are playing an increasingly important role in biomedical applications, where minimally invasive methods are being developed that require imperceptible tissue penetration and drug delivery. To improve the integration of MNs in microelectromechanical devices, a high-resolution 3D printing technique is implemented. A reservoir with an array of hollow MNs is produced. The flow rate through the MNs is simulated and measured experimentally. The mechanical properties of the 3D printed material, such as elasticity modulus and yield strength, are investigated as functions of printing parameters, reaching maximum values of 1750.7 and $101.8 \mathrm{MPa}$, respectively. Analytical estimation of the MN buckling, fracture, and skin penetration forces is presented. Penetration tests of MNs into a skin-like material are conducted, where the piercing force ranges from 0.095 to $0.115 \mathrm{~N}$, confirming sufficient stability of MNs. Furthermore, 200 and $400 \mu \mathrm{m}$-long $\mathrm{MN}$ arrays are used to successfully pierce and deliver into mouse skin with an average penetration depth of 100 and $180 \mu \mathrm{m}$, respectively. A biocompatibility assessment is performed, showing a high viability of HCT 116 cells cultured on top of the MN's material, making the developed MNs a very attractive solution for many biomedical applications.

\section{Introduction}

There is an increasing number of biomedical challenges that require pushing the limits of microtechnologies to enhance and create new tools for diagnostic and therapeutic purposes. This is partially a result of a broad shift from conventional surgical procedures that carry more risks toward less-invasive and patientspecific options. For example, to circumvent the need for ocular

K. Moussi, A. Bukhamsin, Dr. J. Kose

Computer, Electrical and Mathematical Sciences and Engineering Division (CEMSE)

4700 King Abdullah University of Science and Technology (KAUST)

Thuwal 23955, Kingdom of Saudi Arabia

E-mail: jurgen.kosel@kaust.edu.sa

T. Hidalgo

Biological and Environmental Science and Engineering Division (BESE)

4700 King Abdullah University of Science and Technology (KAUST)

Thuwal 23955, Kingdom of Saudi Arabia

The ORCID identification number(s) for the author(s) of this article can be found under https://doi.org/10.1002/adem.201901358.

(C) 2019 The Authors. Published by WILEY-VCH Verlag GmbH \& Co. KGaA, Weinheim. This is an open access article under the terms of the Creative Commons Attribution License, which permits use, distribution and reproduction in any medium, provided the original work is properly cited.

DOI: 10.1002/adem.201901358 injections that are needed to treat many eye conditions, minimally invasive microneedles (MNs) were developed for ocular implants that allow for controlled drug release directly into the eye. ${ }^{[1]}$ Another critical example is the glucose monitoring for diabetic patients, where the continuous diagnosis and insulin injection are stringent. ${ }^{[2]}$ Drugs are commonly administered either orally or via injections, but these routes have significant limitations. Moreover, both routes are also dose limited, as the required dose cannot be administered immediately, due to deleterious side effects associated with the high initial concentration of the drug. Therefore, multiple administrations of the drug are required to achieve the desired effect. This has generated keen interest in exploring alternative routes of delivery such as transdermal delivery, as the skin offers a large and painless interface for drug delivery, ${ }^{[3]}$ in addition to the localized drug delivery inside the body. ${ }^{[4-6]}$

In this regard, recent advancements in microtechnology have helped to make remarkable progress in health-care monitoring and disease therapy. Examples are miniaturized laboratories (Lab-on-Chip, LoC) and microsystems able to sense, diagnose, and deliver a drug to the specific target region. ${ }^{[-9]}$ In this context, the implementation of MNs for drug delivery is one of the most prominent developments in the drug administration field, ${ }^{[10-12]}$ due to their painless and minimally invasive operation. Consequently, MNs have been investigated extensively, involving different materials, such as silicon, ${ }^{[13-16]}$ glass, ${ }^{[17]}$ polymer, ${ }^{[18,19]}$ and metals, ${ }^{[11,20-22]}$ different designs (solid, coated, dissolvable, hollow, etc.), and fabrication techniques. Hollow MNs are of particular interest for their continuous flow and a controllable dose of drug delivery. ${ }^{[14-17]}$ Only in a few works, MNs were integrated into a complex microsystem ${ }^{[23,24]}$ and mostly considered as a standalone device. ${ }^{[13-21]}$

MNs have been previously fabricated using isotropic chemical etching, ${ }^{[15,16]}$ micromolding, ${ }^{[19-22]}$ polysilicon micromolding, ${ }^{[13]}$ and lithography, electroplating, and molding (LIGA). ${ }^{[25]}$ Despite their perceived advantages and applications, MNs are yet to see an extensive pervasion in clinical settings. In part, this is due to the complexities associated with the microfabrication techniques used for the development of MNs, which are often multistep, labor intensive, and require expensive cleanroom equipment. In addition, complications arise from the handling and attachment of MNs fabricated with a specific process to a device or structure fabricated with another process. 
With the advent of methods to print beyond the optical diffraction limit, 3D printing, also known as additive manufacturing, has become a viable option for the fabrication of MNs. It has evolved via the introduction of different technologies such as stereolithography (SLA), fused deposition modeling (FDM), and two-photon polymerization (TPP). SLA has been used in various works to produce $\mathrm{MNs}$ for transdermal drug delivery, mainly to fabricate plain/solid MNs. ${ }^{[26-30]}$ The delivery method can be either by coating the MNs with the required drug ${ }^{[28]}$ or can be preloaded in the case of biodegradable materials. ${ }^{[29]}$ This is not adequate for applications which demand larger delivery doses over a short time interval or require the continuous constant release of therapeutic agents through the skin. Nevertheless, Farias et al. were able to fabricate a 3D printed hollow MN device to inject encapsulated epithelial cells for wound-healing applications. ${ }^{[31]}$ To a lesser extent than SLA, the FDM 3D printing technique was used recently to fabricate biodegradable polymer MNs for transdermal drug delivery. ${ }^{[32]}$ Adoption of these technologies enables control over desired MNs geometry while not compromising neither the resolution nor the aspect ratio. Furthermore, 3D printing may facilitate easy customization of transdermal drug delivery systems to accommodate for factors that influence delivery such as differences in skin thickness and hydration. A more exciting approach, yet underexplored, reported by Ovsianikov and coworkers, relies on the use of TPP to fabricate hollow hypodermic MNs for transdermal delivery. Using such an array could allow for direct interfacing of the MNs array with a refillable drug reservoir for continuous operation. In this study, we explore the potential of using TPP to fabricate an array of hollow hypodermic MNs attached to a drug reservoir. The integrated nature of such a design allows for interfacing with larger delivery loads. This approach has already been implemented using standard processing techniques of silicon microfabrication ${ }^{[33]}$ and using metal electrodeposition of a patterned sacrificial mold ${ }^{[34]}$ to create hollow MNs with an attached reservoir. However, these processing techniques suffer from the same complexities as mentioned earlier and afford limited control over the MNs geometry and aspect ratio. Thus, with the advent of TPP, it is now feasible to 3D print microstructures having dimensions in the millimeter scale while providing feature sizes with micrometer resolution. ${ }^{[35,36]}$

Hence, in this work, the TPP 3D printing technique is used to fabricate various designs of hollow MNs, made of IP-S photoresist, with an aspect ratio (height/outer diameter of the $\mathrm{MN}$ tip) ranging from 1 to 20. The design, fabrication, fluidic, and mechanical properties are studied, and the biocompatibility is investigated. The MNs are combined with the reservoir, making it suitable for implanting inside the body, transdermal sampling, or drug delivery applications. We demonstrate a fabrication process that allows producing hollow MNs directly connected to a reservoir, which is used to load or extract fluid, in a single fabrication step. The MNs inner diameter and height presented range from 80 to $120 \mu \mathrm{m}$ and from 200 to $400 \mu \mathrm{m}$, respectively. The MNs wall thickness at the tip is $5 \mu \mathrm{m}$. However, the process is characterized by a high degree of flexibility with respect to the resolution and design parameters. The buckling, yield strength, and skin penetration forces were analyzed theoretically and experimentally. Furthermore, the penetration and delivery capabilities of the MNs were tested on polydimethylsiloxane (PDMS) and mouse skin.
An empirical fluidic characterization of the fabricated MNs establishes a link between the flow rate, geometry, and the number of needle bores. A cytotoxicity assay was used to study the viability of cells grown on IP-S photoresist and evaluate the toxic potential of the MNs for biomedical applications.

\section{Theoretical Study}

\subsection{Estimation of the Buckling, Fracture, and Penetration Forces}

MNs used for transdermal delivery have to overcome the skin's mechanical resistance by piercing the stratum corneum and penetrate up to the dermis layer without mechanical failure. In this regard, the prediction of the forces applied to the MNs is a critical design aspect. Since the MNs feature high aspect ratios and low tapering angles, they are mainly prone to buckling and fracture. The yield failure known as the fracture is due to an applied load higher than the yield strength of the MN material, whereas the buckling failure leads to deformation of the MNs into an arched shape. To predict the buckling force applied on the MNs, we used the analytical model derived by Kim et al. for the fixed-free tapered hollow truncated cone structure. ${ }^{[22]}$ The final form of the equation is presented in Equation $S(1)$, Supporting Information. The estimation of the fracture force was based on the assumptions that the failure or fracture of the $\mathrm{MN}$ is caused by axial forces applied to the MN tip, which means that shear forces are neglected and that the MN fracture is mainly due to an applied pressure higher than the ultimate stress of the material. Based on these assumptions, the MN fracture force can be modeled by Equation S(2), Supporting Information. The MN penetration force into the human skin has been investigated thoroughly by Davis et al. ${ }^{[37]}$ and Khanna et al., ${ }^{[38]}$ where they measured the MN insertion force into the human skin and were able to determine the skin toughness from the experimental data. To predict the required force for a MN to pierce the human skin, Davis et al. ${ }^{[37]}$ have developed an empirical expression of the insertion force based on the puncture fracture toughness $G_{\mathrm{p}}$ and the $\mathrm{MN}$ geometry (Equation $\mathrm{S}(3)$, Supporting Information).

The fracture toughness $G_{\mathrm{p}}$ of the human skin ranges from $G_{\mathrm{p} 1}=30.1 \mathrm{~kJ} \mathrm{~m}^{-2}$ (hard skin) to $G_{\mathrm{p} 2}=20 \mathrm{~kJ} \mathrm{~m}^{-2}$ (soft skin), as reported by Davis et al. and Khanna et al., respectively. ${ }^{[37,38]}$

\subsection{Flow Rate Estimation}

The finite element method (FEM) was used to simulate the volumetric flow rate for the different designs (Table 1), and the modeling was implemented in SolidWorks Flow Simulation (Dassault Systèmes SolidWorks Corporation, MA, USA). The software solves Navier-Stokes equations in a parallelepiped computational domain with the finite volume method. The flow type was set to be laminar and turbulent with water as an incompressible fluid. The boundary conditions consisted of atmospheric pressure at the MNs outlet and a variable input pressure at the reservoir inlet of 3$10 \mathrm{kPa}$. The flow velocity profile for sample S1 $(8 \mathrm{MNs} 80 \mu \mathrm{m} \varnothing-$ $200 \mu \mathrm{m}$ long) at an applied pressure of $10 \mathrm{kPa}$ can be found in Figure S1, Supporting Information. 
Table 1. Dimensions of the 3D printed specimens used for the fluidic characterization.

\begin{tabular}{lcccc}
\hline Sample & S1 & S2 & S3 & S4 \\
\hline MN inner diameter & $80 \mu \mathrm{m}$ & $120 \mu \mathrm{m}$ & $80 \mu \mathrm{m}$ & $80 \mu \mathrm{m}$ \\
MN height & $200 \mu \mathrm{m}$ & $200 \mu \mathrm{m}$ & $400 \mu \mathrm{m}$ & $200 \mu \mathrm{m}$ \\
MN number & 8 & 8 & 8 & 4 \\
3D model & & & &
\end{tabular}

\section{Experimental Section}

\subsection{Materials Preparation}

The microchip design consisted of a drug reservoir with an array of $\mathrm{MNs}$ on top of it. The reservoir dimensions were $2 \times 1 \times 1 \mathrm{~mm}^{3}$. Four different designs were fabricated (Table 1 ).

Sample S1 had an array of eight MNs arranged in two rows of four MNs each, where the MNs' diameter and height were 80 and $400 \mu \mathrm{m}$, respectively. The other designs shown in Table 1 had either a larger $\mathrm{MN}$ diameter (S2), a longer MN height (S3), or a lesser number of MNs (S4) compared to (S1). Before 3D printing, the SLA file of each design was converted into a general writing language script using Describe software (Nanoscribe $\mathrm{GmbH}$, Germany). The slicing distance, which is the distance between the layers in the vertical direction, was set to $2 \mu \mathrm{m}$. The hatching distance, which is the distance between two lines of the laser beam in the horizontal plane, was set to $2 \mu \mathrm{m}$. Both parameters (slicing/hatching) were chosen after an optimization process to fabricate a robust structure in a short period of time. A summary of the optimal parameters used to convert the SLA files into a 3D printable format is shown in Table S1, Supporting Information.

The $3 \mathrm{D}$ printing fabrication process started with a $500 \mu \mathrm{m}$ thick single-side polished silicon substrate. Prior to the 3D printing step, an elliptical/rectangular void (minor axis $0.8 \mathrm{~mm}$ and major axis $1.5 \mathrm{~mm}$ ) was etched through the silicon substrate using a $50 \mathrm{~W}$ fiber laser (PLS6MW, Universal Laser Systems $\mathrm{GmbH}$, Vienna, Austria) with $1.06 \mu \mathrm{m}$ wavelength (Figure S2a, Supporting Information). Then, the substrate was cleaned in an ultrasonication bath of acetone and isopropyl alcohol. Subsequently, it was washed with deionized (DI) water and dried using a gentle stream of nitrogen gas. IP-S photoresist (Nanoscribe $\mathrm{GmbH}$, Germany) was then drop cast on the center of the silicon substrate (Figure S2b, Supporting Information), on top of the elliptical hole, and loaded into the Nanoscribe Photonic Professional GT laser lithography system (Nanoscribe $\mathrm{GmbH}$, Germany). ${ }^{[39]}$ The designed structure was printed layer by layer in a dip-in laser lithography configuration. The objective lens $(25 \times$ magnifications and NA $=0.8)$ was immersed in the resist and focused on the silicon interface, then, positioned at the void center. IP-S was chosen for its low shrinkage effect, smooth surfaces, and ability to print feature size ranging from the submicron to the millimeter scale. ${ }^{[39]}$ Polymerization of the photoresist was induced by the laser at $780 \mathrm{~nm}$ wavelength, $100 \mathrm{~mW}$ power, and $50 \mathrm{~mm} \mathrm{~s}^{-1}$ scan speed. Following the printing process, the 3D printed assembly (reservoir and $\mathrm{MNs}$ ) was developed by immersion in mr-DEV 600 (microresist technology $\mathrm{GmbH}$, Germany) for $10 \mathrm{~min}$ to remove the unpolymerized excess of resist (Figure S2d,e, Supporting Information). Then, to clear the MNs channels, the sample was immersed again in the developing solution under vacuum for $15 \mathrm{~min}$. Subsequently, it was immersed in isopropanol (IPA) for an additional $5 \mathrm{~min}$ to remove residual photoresist and dilute the remaining developing solution. Finally, the sample was dried with a gentle stream of nitrogen gas (Figure S2f, Supporting Information). This process was applied to fabricate the four different samples, i.e., S1, S2, S3, and S4 (Table 1).

For the penetration tests, only the MNs were of interest. Using the same substrate $(500 \mu \mathrm{m}$-thick, single-side polished silicon substrate), but this time without etching a void through the silicon, MNs were made of IP-S photoresist. The MNs geometry featured a tapered angle of $5^{\circ}$, an inner diameter of $30 \mu \mathrm{m}$, an outer tip diameter of $50 \mu \mathrm{m}$, and a height of 1000 and $200 \mu \mathrm{m}$, respectively. Except for the slicing/hatching distance that changed to $1 \mu \mathrm{m}$, the same printing parameters mentioned in the fabrication of the assembly reservoir and MNs were used. Then, MNs were developed by immersion in mr-DEV 600 for $10 \mathrm{~min}$, followed by immersion in IPA for $5 \mathrm{~min}$, and dried with a gentle stream of nitrogen gas.

Scanning electron microscope images of the MNs can be found in Figure S3, Supporting Information.

\subsection{Tensile Test}

The mechanical properties of the IP-S photoresist were determined experimentally by performing a tensile test on samples (Table 2) fabricated with different printing settings. A 5966 Dual Column Electromechanical Testing System (Instron ${ }^{\circledR}$ ) with a load cell of $10 \mathrm{~N}$ was used to determine Young's modulus and yield strength of the material (Figure S4, Supporting Information).

\subsection{MNs Penetration}

To test the MNs on a material that has skin-like mechanical properties, PDMS samples were created with an elasticity modulus 
Table 2. Dimensions of the 3D printed specimens used for tensile mechanical testing.

\begin{tabular}{lccc}
\hline & $\begin{array}{c}\text { Slicing } \\
\text { distance }[\mu \mathrm{m}]\end{array}$ & $\begin{array}{c}\text { Hatching } \\
\text { distance }[\mu \mathrm{m}]\end{array}$ & $\begin{array}{c}\text { Dimensions } \\
{[w \times t \times l]\left[\mu \mathrm{m}^{3}\right]}\end{array}$ \\
\hline Specimen 1 & 1 & 1 & $300 \times 300 \times 2000$ \\
Specimen 2 & 2 & 2 & $300 \times 300 \times 2000$ \\
\hline
\end{tabular}

equal to or higher than the one of the human skin. The mechanical properties of human skin were investigated in vivo by Liang and Boppart ${ }^{[40]}$ in different locations of the human body and dehydration levels of the skin. They found that the elasticity modulus varies from 0.1 to $0.3 \mathrm{MPa}$. In the case of PDMS, the elasticity modulus is linearly dependent on the crosslinking ratio ${ }^{[41]}$ (from 5:1 to 33:1), with values between 3.6 and $56 \mathrm{MPa}$, respectively ${ }^{[41]}$ We used a crosslinking ratio of 10:1 (base/curing agent) to prepare PDMS skins (Sylgard 184 Silicone Elastomer, Dow Corning Corp., Midland, MI, USA), corresponding to an elasticity modulus of $2.6 \mathrm{MPa}$, which is about an order of magnitude higher than the Young's modulus of human skin. Using the Electromechanical Testing System, a single MN was attached to the indenter, and a PDMS skin was placed on top of a support, as shown in Figure 1. The PDMS skins were prepared by drop casting with 700 and $160 \mu \mathrm{m}$ of thickness, depending on the height of the MN (1000 and $200 \mu \mathrm{m}$ long, respectively). The insertion rate was $5 \mu \mathrm{m} \mathrm{s}^{-1}$.

To evaluate the penetration depth of the MNs array and validate delivery of a liquid solution into the skin, a fluorescent dye was injected through the MNs into mouse skin (Figure 1b). Before testing, a hollow (1 $\mathrm{mm}$ in diameter) acrylic sheet (10 by 10 and $1 \mathrm{~mm}$ thickness) was cut using a laser cutter (Universal PLS6.75 $10.6 \mu \mathrm{m} \mathrm{CO}$ ). Then, a 19G blunt tip needle and the MNs array were glued to the back and front sides, respectively, of the acrylic substrate using super glue, as shown in the inset of Figure $1 \mathrm{~b}$. With this assembly, the MN array was applied manually on the back and chest of a euthanized female nude mouse (10 months old, CD-1 nude mouse, Charles River laboratories). Using a $1 \mathrm{~mL}$ syringe connected to the $19 \mathrm{G}$ needle, fluorescein isothiocyanate (FITC) (Sigma Aldrich, USA) dye was injected. The mouse skin was then excised and imaged using a Leica SP8 inverted confocal microscope (Leica, Germany) with a $10 \times$ objective. The MN samples used in the mouse skin penetration experiment were S1 and S3 (200 and $400 \mu \mathrm{m}$ long, respectively) (Table 1). For each MN type, five samples were tested.

\subsection{Flow Rate Measurement}

The custom test setup was composed of a pressure flow controller Elveflow OB1 (ELVESYS, Paris, France), a Mariotte bottle, a weigh boat, a digital scale (1 $\mathrm{mg}$ resolution), FEP tubing, a digital camera, and a 3D printed sample, as shown in Figure S5, Supporting Information.

Before testing, a 19G blunt tip needle was glued on the back side of the silicon wafer. The dispensing needle was used to connect the 3D printed reservoir to the flexible tube (inner diameter of $1 \mathrm{~mm}$ ). The Mariotte bottle was filled with DI water and sealed. After connecting all the components in the testing setup, the pressure controller was monitored by a computer to supply a constant pressure ranging from 3 to $10 \mathrm{kPa}$ with a step of $1 \mathrm{kPa}$ for each measurement. The DI water flowing through the MNs was collected in a weigh boat on top of the digital balance. The fluidic mass flow rate was measured by recording the mass variation over time using a digital camera. Then, the mass flow rate was converted to a volumetric flow rate by dividing it by the mass density of the fluid. The measurement was repeated three times for each pressure increment in addition to testing two identical 3D printed samples for each model (two samples of each S1, S2, S3, and S4 were tested).

\subsection{Cytotoxicity Test}

HCT 116 cells were plated onto cell culture flasks (Corning Inc., Corning, NY) with McCoy's 5A modified medium (ThermoFisher Scientific, Massachusetts, USA), supplemented with fetal bovine serum up to a concentration of $10 \%$, and incubated at $37^{\circ} \mathrm{C}$ in a humidified atmosphere chamber with $5 \% \mathrm{CO}_{2}$. When cells were 80-90\% confluent, old medium was aspirated, and cells were washed with 1X Dulbecco's phosphate-buffered saline solution (GIBCO, ThermoFisher Scientific, Massachusetts, USA) and detached with ACCUTASE ${ }^{\mathrm{TM}}$ (StemCell Technologies Inc., BC, Canada). McCoy's 5A modified medium was added to inactivate (a)

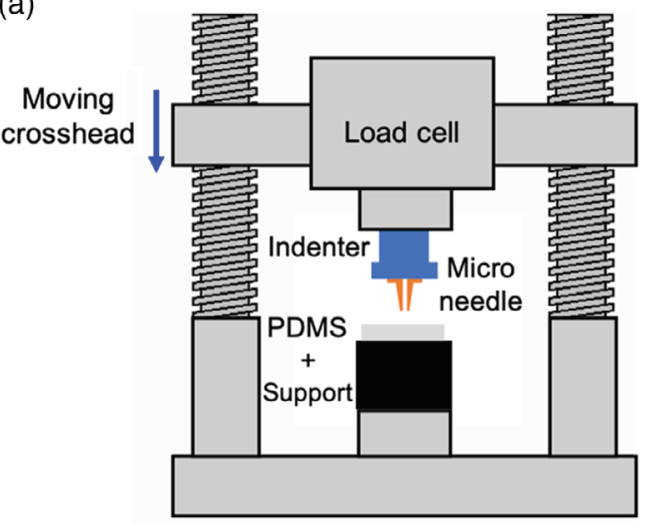

(b)

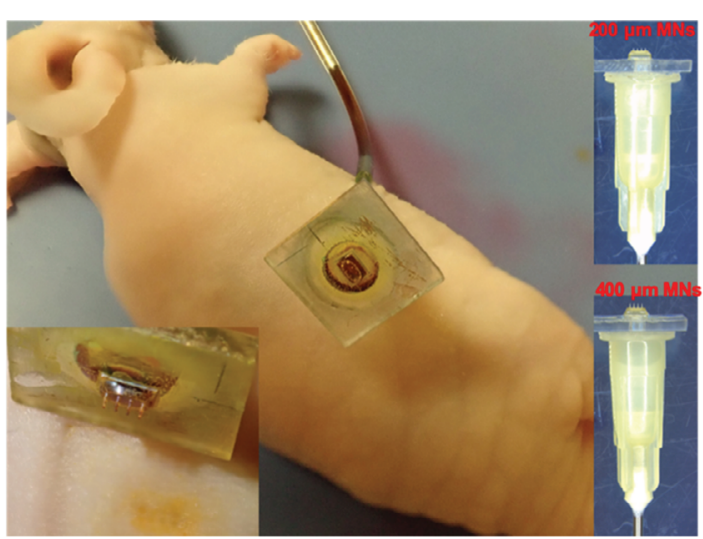

Figure 1. MN penetration testing. a) A schematic view of the setup used for PDMS skin-like samples. b) In vitro skin piercing experiment. 
the ACCUTASE after cell detachment. The cells were centrifuged, resuspended in the culture medium, and seeded onto 96-well plates and 24-well plates (Corning Inc., Corning, NY) for the cytotoxicity assays. Discs of IP-S resin were made by drop casting an amount of the resin into a PDMS template having circular cavities with of $6 \mathrm{~mm}$ diameter and $1 \mathrm{~mm}$ depth, which was cured in an oven at $200^{\circ} \mathrm{C}$ for $30 \mathrm{~min}$.

HCT 116 cells were plated in 96-well plates at 10000 cells/well with $100 \mu \mathrm{L}$ of medium and left in an incubator at $37^{\circ} \mathrm{C}$ with $95 \%$ relative humidity and $5 \% \mathrm{CO}_{2}$ for $24 \mathrm{~h}$ to create a monolayer. After $24 \mathrm{~h}$, old medium was aspirated from the wells and replaced with the medium used as extract vehicle. Cells were left in an incubator for $24 \mathrm{~h}$ with the extract vehicle and, afterward, the PrestoBlue assay was performed. The same procedure was followed to test cell viability with extract vehicle contact after $48 \mathrm{~h}$. For the direct contact experiments, the preparation for testing was also the aforementioned one but, after creating the monolayer of cells, the medium was replaced, and the photoresist discs were put in contact with the monolayer, both for 24 and $48 \mathrm{~h}$. For the 24-well plates, cells were plated at 50000 cells/well with $500 \mu \mathrm{L}$ of medium. Following ISO 10993 for the biological evaluation of medical devices, ${ }^{[42]}$ three types of cytotoxicity assays were performed. The first assay consisted of placing a piece of the tested material in direct contact with the cells while they were growing. The second and third assays required the exposure of the material to the medium in which the cells were grown so that it could be used to grow cells. The extractions were conducted both in a mixer and in an incubator at $37^{\circ} \mathrm{C}$, according to the surface area-based ratios set in ISO 10993-12, ${ }^{[43]}$ in sealed vessels for $24 \mathrm{~h}$. A piece of $0.75 \mathrm{~cm}^{2}$ of hardened photoresist was put in contact with the extraction vehicle (McCoy's 5A modified medium, supplemented with fetal bovine serum up to a concentration of $10 \%$ ) in a chemically inert container for $24 \mathrm{~h}$ in a mixer at $500 \mathrm{rpm}$ and $37^{\circ} \mathrm{C}$. The other type of extraction was done by placing both the piece of photoresist and the
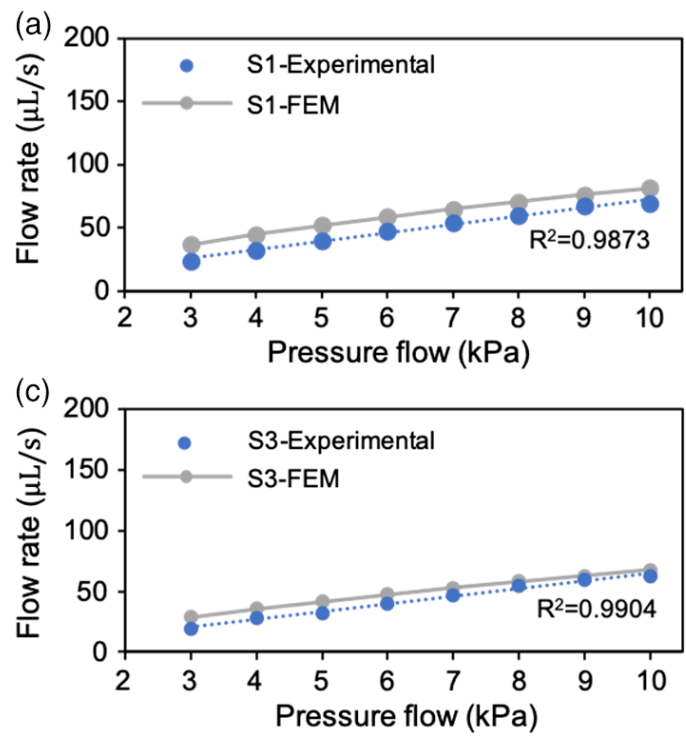

extraction vehicle in an incubator at $37^{\circ} \mathrm{C}$ with $5 \% \mathrm{CO}_{2}$. For cell viability visualization, cells were washed with phosphatebuffered saline (PBS) $1 \mathrm{X}$ and incubated in PBS $1 \mathrm{X}$ with $4 \mu \mathrm{M}$ ethidium homodimer and $2 \mu \mathrm{M}$ calcein $\mathrm{AM}$ for $30 \mathrm{~min}$, after which the samples were imaged using the laser scanning microscope 710 (Carl Zeiss Microscopy GmbH, Germany).

\section{Results and Discussion}

\subsection{Flow Rate}

The flow rate as a function of pressure is shown for all samples in Figure 2. All experimental results showed an excellent linear fit (R2 ranging between 0.99 and 0.98 ) and agreed well with the FEM simulation (Figure 2). This linearity of the experimental result suggests a fully developed laminar flow through the needle bores, which is also corroborated by the calculated Reynolds numbers, which ranged between 30 and 230. The experimental flow rates were lower than the results found by FEM. This is attributed to the existence of losses occurring before the reservoir and MNs caused by the Mariotte bottle, the pipes, and connections. This is also consistent with previous reports by Takei et al. ${ }^{[14]}$ These losses were not considered in the FEM model. The experimental flow rates through sample S1 (Figure 2a), which has $8 \mathrm{MNs}$ with a diameter of $80 \mu \mathrm{m}$ and a shaft length of $200 \mu \mathrm{m}$, are approximately double that of sample S4 (Figure 2d), which has four needles of the same dimensions, through the entire pressure range. As suggested by Strambini et al., ${ }^{[23]}$ the experimentally acquired flow rates support the hypothesis that the aggregate flow through the array is a result of independent flow rates through $\mathrm{N}$ identical MNs that experience the same drop in pressure. The spacing of the needles in the array minimizes cross influence between the independent flows. As a result, the flow profile can be modulated by maintaining the
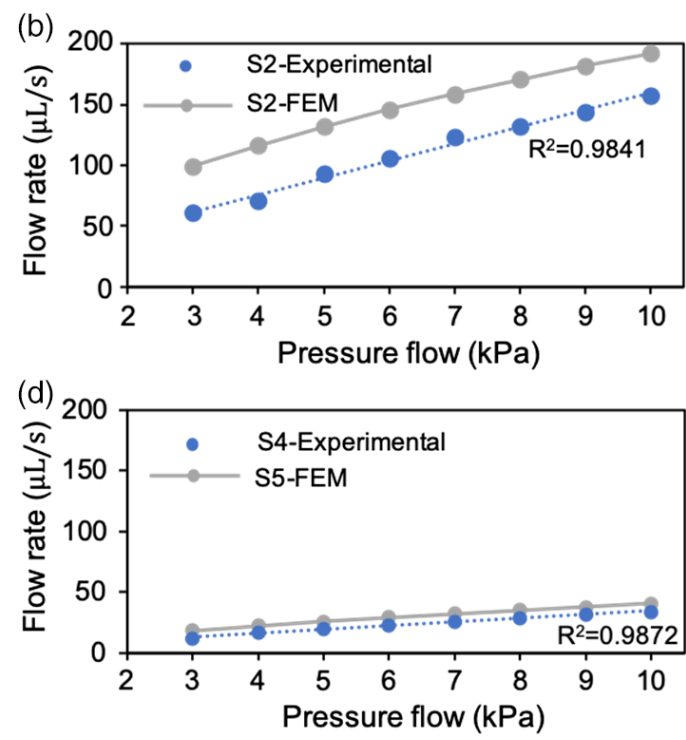

Figure 2. The flow rate through 3D printed MNs for four different geometries. a) Sample S1 featuring eight MNs with $80 \mu \mathrm{m}$ diameter and $200 \mu \mathrm{m}$ height. b) S2 featuring eight MNs with $120 \mu \mathrm{m}$ diameter and $200 \mu \mathrm{m}$ height. c) S3 featuring eight MNs with $80 \mu \mathrm{m}$ diameter and $400 \mu \mathrm{m}$ height. d) S4 featuring four MNs with $80 \mu \mathrm{m}$ diameter and $200 \mu \mathrm{m}$ height $(n=2$, mean \pm standard error (SE)). 
spacing and adjusting the needle count. The impact of bore radius and shaft length on the flow rate is notable when comparing sample S1 (Figure 2a) to sample S2 (Figure 2b) and sample S3 (Figure 2c), respectively. Increasing the radius from $80 \mu \mathrm{m}$ (S1) to $120 \mu \mathrm{m}$ (S2) nearly doubled the flow rate. Similarly, increasing the shaft length from $200 \mu \mathrm{m}$ (S1) to $400 \mu \mathrm{m}$ (S3) reduced the flow rate by $33 \%$.

\subsection{Mechanical Properties}

The tensile test on the 3D printed IP-S bars allowed the determination of the stress-strain curve (Figure 3) from which the elasticity modulus and the yield strength were extracted. The sample with $1 \mu \mathrm{m}$ of slicing/hatching distances has stronger mechanical properties. The elasticity modulus and yield strength are $1740 \pm 15$ and $100 \pm 2.8 \mathrm{MPa}$, respectively, for the samples with $1 \mu \mathrm{m}$ of slicing/hatching distances, and they are equal to $867.27 \pm 27.04$ and $64.58 \pm 5.74 \mathrm{MPa}$, respectively, for the sample with $2 \mu \mathrm{m}$ of slicing/hatching distances. Decreasing the slicing and hatching distances resulted in denser structures, which had about two times stronger mechanical properties. This suggests that the material strength and elasticity can be tailored to intermediate properties by modifying the printing parameters, particularly the slicing and hatching distances.

\subsection{Buckling, Fracture, and Penetration Forces}

The buckling forces were estimated for two different $\mathrm{MN}$ heights, 200 and $1000 \mu \mathrm{m}$, represented by $F_{\mathrm{b} 200}$ and $F_{\mathrm{b} 1000}$, respectively, as shown in Figure 4. The skin puncturing force was assessed for the two reported fracture toughness limits $G_{\mathrm{p} 1}$ and $G_{\mathrm{p} 2}$, which correspond to a hard and soft skin and are shown in Figure 4 by the dashed lines for $F_{1}$ piercing and $F_{2}$ piercing, respectively. Generally, with increasing MN outer diameter, the buckling force increases. The buckling effect is stronger for the $1000 \mu \mathrm{m}$-long MN regardless of the MN diameter. The $1000 \mu \mathrm{m}$-long MN will always buckle before a fracture occurs, whereas in case of the $200 \mu \mathrm{m}$-long $\mathrm{MN}$, the fracture

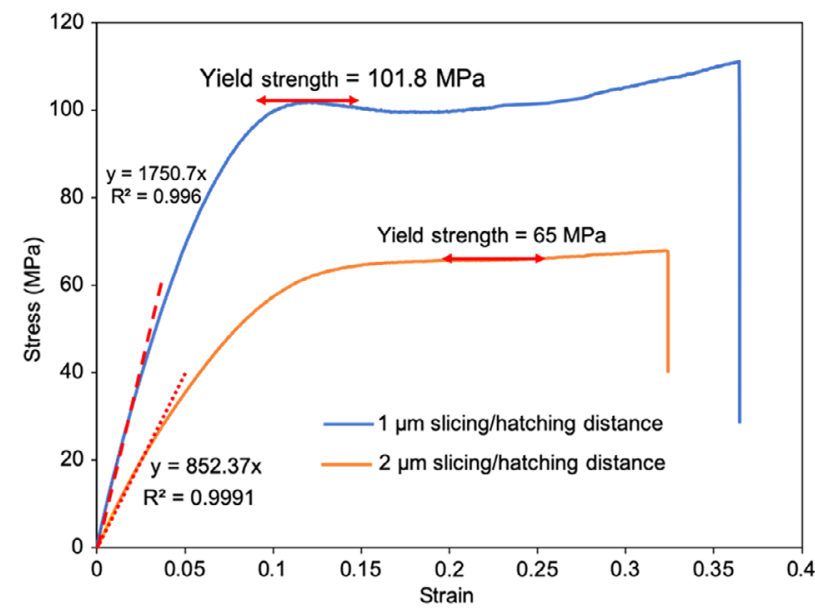

Figure 3. Stress-strain curve of two 3D printed IP-S bars. The blue curve corresponds to a slicing/hatching distance of $1 \mu \mathrm{m}$. The orange curve corresponds to a slicing/hatching distance of $2 \mu \mathrm{m}$.

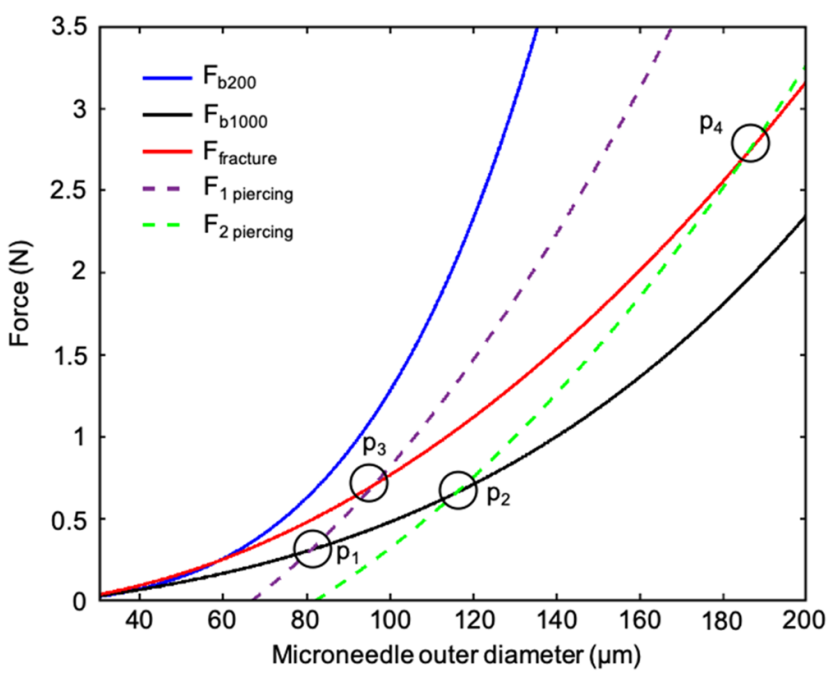

Figure 4. Theoretical evaluation of the buckling, fracture, and penetration forces. $F_{\mathrm{b} 200}$ and $F_{\mathrm{b} 1000}$ are the buckling forces of the 200 and $1000 \mu \mathrm{m}$ long MNs, respectively. $F_{\text {fracture }}$ is the MN's tip fracture force. The dashed lines $F_{1}$ piercing and $F_{2}$ piercing denote the skin piercing forces, for $G_{p 1}$ and $G_{\mathrm{p} 2}$, respectively. p1 $(80 \mu \mathrm{m}, 0.3 \mathrm{~N}) ; \mathrm{p} 2(115 \mu \mathrm{m}, 0.64 \mathrm{~N}) ; \mathrm{p} 3(95 \mu \mathrm{m}$, $0.67 \mathrm{~N})$; and $\mathrm{p} 4(180 \mu \mathrm{m}, 2.5 \mathrm{~N})$ are critical points in the graph.

force is higher than the buckling force only for an outer diameter less than $60 \mu \mathrm{m}$. For bigger diameters, the MN will face fracture before buckling. The minimum required force to puncture the skin for a MN with an outer tip diameter less than $80 \mu \mathrm{m}$ is estimated to be less than $0.01 \mathrm{~N}$ based on the theoretical analysis (Figure 4), which confirms the results found by Roxhed et al ${ }^{[4]}$ In case of the $1000 \mu \mathrm{m}$-long MN, hard skin can be penetrated without buckling, when the $\mathrm{MN}$ diameter is smaller than $80 \mu \mathrm{m}$ (critical point p1), whereas soft skin can be penetrated when the diameter is smaller than $115 \mu \mathrm{m}$ (critical point p2).

Nevertheless, the penetration into the skin is still possible by applying additional force, while not reaching the fracture force limits ( $\mathrm{p} 3$ for the hard skin and $\mathrm{p} 4$ for the soft skin). Therefore, for a $1000 \mu \mathrm{m}$-long IP-S 3D printed MN, the maximum outer diameter has to be less than $80 \mu \mathrm{m}$ to puncture the human skin without any mechanical failure (considering the hard skin). Moreover, regardless of the MN height, the MN tip diameter has to be less than either 95 or $180 \mu \mathrm{m}$ based on the type of skin; otherwise, the MN will break before penetrating the skin. The skin penetration of the $200 \mu \mathrm{m}$-long $\mathrm{MN}$ is limited by fracture only, with the same values as the $1000 \mu \mathrm{m}$-long $\mathrm{MN}$.

\subsection{MN Penetration Test}

As shown in Figure 5, both MNs (200 and $1000 \mu \mathrm{m}$ ) were able to puncture and penetrate the PDMS layers without mechanical failure. The $200 \mu \mathrm{m}$-long $\mathrm{MN}$ penetrates the $160 \mu \mathrm{m}$-thick PDMS layer at an applied force of $0.095 \mathrm{~N}$ and after displacement of about $118 \mu \mathrm{m}$ (Figure 5a). Before puncturing, the PDMS layer was deformed and buckled, due to its elasticity. Similarly, the $1000 \mu \mathrm{m}$-long MN penetrates the $700 \mu \mathrm{m}$-thick PDMS layer at an applied force of $0.115 \mathrm{~N}$ and after displacement of about $480 \mu \mathrm{m}$ (Figure $5 \mathrm{~b}$ ). After puncturing, the force remains constant 

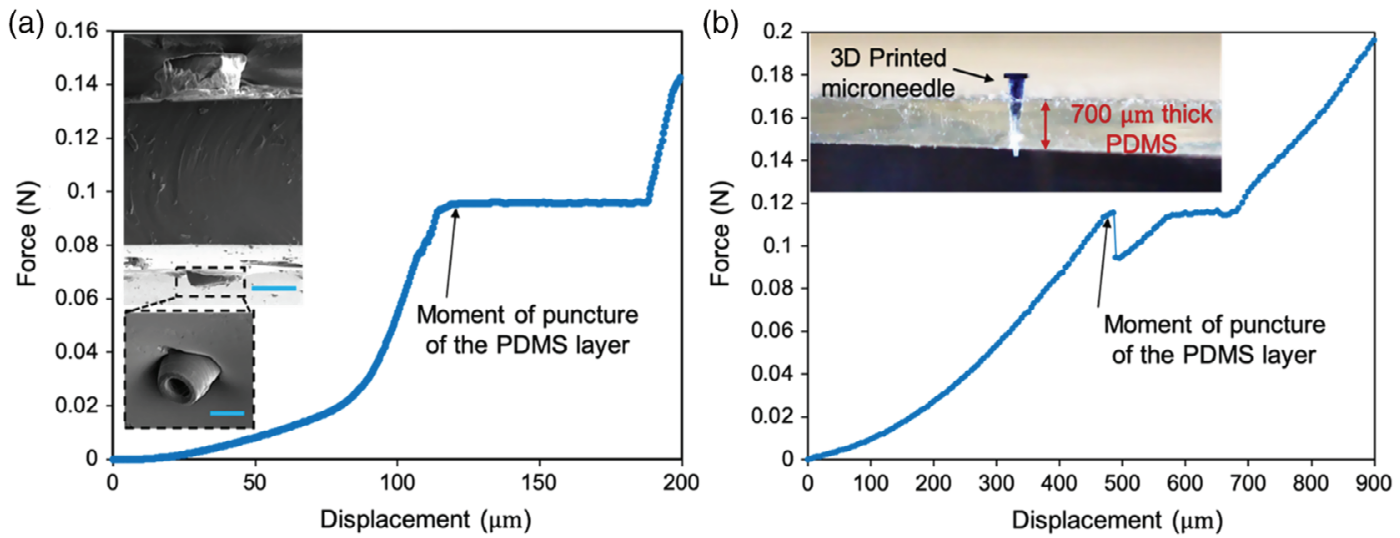

Figure 5. Measurement of the insertion force of the a) $200 \mu \mathrm{m}$ and b) $1000 \mu \mathrm{m}$-long MNs into PDMS 10:1. Inset in (a): photograph of the $200 \mu \mathrm{m}$-long MN penetrating a $160 \mu \mathrm{m}$-thick PDMS layer with a zoom on the tip. Inset in (b): photograph of the $1000 \mu \mathrm{m}$-long MN penetrating a $700 \mu \mathrm{m}$-thick PDMS layer. Scale bar: $50 \mu \mathrm{m}$.

(plateau region in both curves in Figure 5) until it increases again, due to the direct contact of the MNs with the support under the PDMS layer. Although the tip geometry is similar for the two MNs, the piercing forces were slightly different $(0.095$ and $0.115 \mathrm{~N}$ for the 200 and $1000 \mu \mathrm{m}$-long MNs, respectively) due to the difference in the PDMS layer thickness (160 and $700 \mu \mathrm{m}$ for the 200 and $1000 \mu \mathrm{m}$-long MNs, respectively). Khanna et al. studied the penetration force of an array of $4 \times 4$ silicon MNs with 160 and $85 \mu \mathrm{m}$ of outer and inner diameters, respectively, on a $760 \mu \mathrm{m}$-thick human cadaver skin harvested from the back. ${ }^{[38]}$ The insertion force for the set of $16 \mathrm{MNs}$ was $1.8 \mathrm{~N}$. Assuming an equal distribution of the force on the set of MNs, the force per single $\mathrm{MN}$ is $0.1125 \mathrm{~N}$, which is in the same order as the values found in our test on the PDMS material. Davis et al. measured the MNs insertion force against the skin of human subjects. The MNs used were $720 \mu \mathrm{m}$ long with a tip radius and wall thickness varying from $30-80$ to $5-58 \mu \mathrm{m}$, respectively. ${ }^{[37]}$ For a comparable MN tip area (about $1300 \mu \mathrm{m}^{2}$ ), the measured insertion force was about $0.1 \mathrm{~N}$ which confirms the insertion forces measured in Figure 5.

The images shown in Figure 6a,b confirm the puncturing of the mouse skin by creation of pores identical to the MNs array. Using confocal microscopy, image stacks were taken from the skin surface down to $100 \mu \mathrm{m}$ (for the $200 \mu \mathrm{m}$-long MNs) and $200 \mu \mathrm{m}$ (for the $400 \mu \mathrm{m}$-long MNs) below the skin with a $20 \mu \mathrm{m}$ step size (Figure S6, Supporting Information). The 3D reconstruction of the z-stack images of the FITC dye signal clearly showed the dye delivery and diffusion into the mouse skin (Figure $6 c, d$ ). (a)

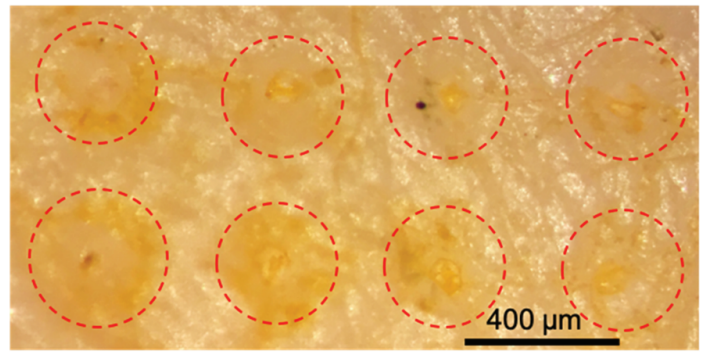

(b)

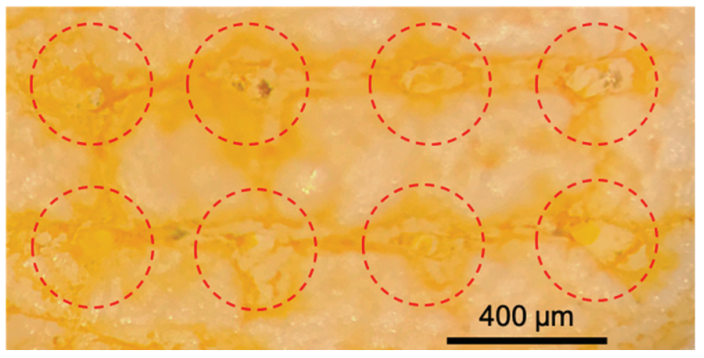

(c)

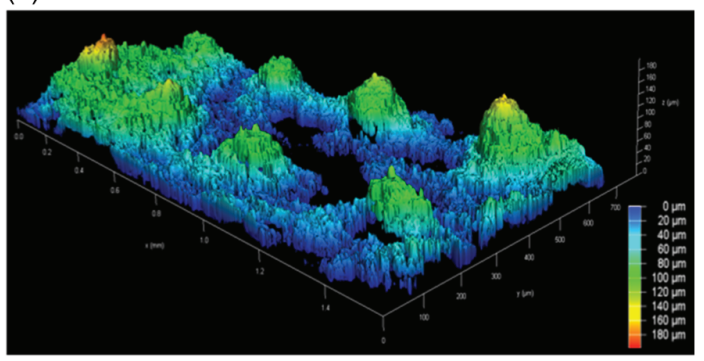

(d)

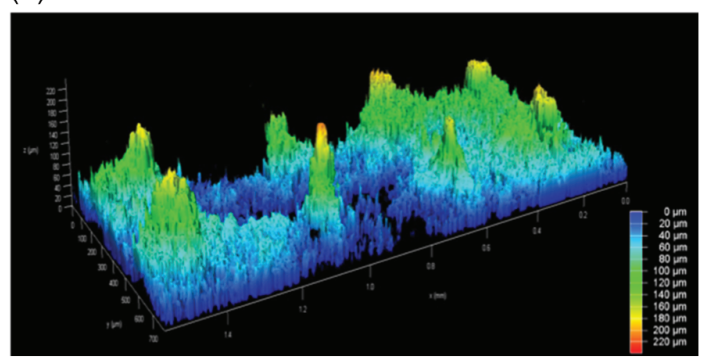

Figure 6. MNs penetration and delivery of FITC dye into mouse skin. a,b) Photograph of the skin after penetration and dye delivery using 200 and $400 \mu \mathrm{m}$-long MN arrays, respectively. c,d) 3D representation of dye delivery in the mouse skin for the 200 and $400 \mu \mathrm{m}$-long $\mathrm{MN}$ arrays, respectively. The blue side $(z=0)$ corresponds to the skin surface, and $z>0$ represents the penetration into the skin. 
As shown in Figure 6c,d, the maximum penetration depth was about 160 and $220 \mu \mathrm{m}$ for the 200 and $400 \mu \mathrm{m}$-long MNs, respectively. However, the average penetration and delivery of the MNs array were at about 100 and $180 \mu \mathrm{m}$ for the 200 and $400 \mu \mathrm{m}$-long MNs, respectively. This result indicates that the delivery and penetration depth are about $50 \%$ of the MNs length. Previous works reported comparable results with a penetration depth of about $30-50 \% .^{[4,45]}$ The presence of dye at the skin surface is mainly due to the overflow of the manual delivery using a syringe, in addition to the irregular shape of the MN-produced pores that allowed leakage to the surface. Similar observations have been reported by Gittard et al., where the pores had irregular shapes, and fluorescent tenting was present in the first $36 \mu \mathrm{m}$ skin depth. ${ }^{[46]}$ As shown in Figure S7, Supporting Information, the $\mathrm{MN}$ array punctured and penetrated the mouse skin without any mechanical damage to the MN tip and shaft.

\subsection{Biocompatibility}

The results of the cytotoxicity test in Figure 7a show a decrease in cell viability of no more than $10 \%$ for cells grown on top of cured IP-S resin for 24 and $48 \mathrm{~h}$. Furthermore, the LIVE/DEAD ${ }^{\text {TM }}$ viability assay confirms the growth of the cells on the resist substrate, as shown in Figure 7b,c, which shows living cells with an insignificant number of dead cell signals. According to ISO 10993-5 (part 8.5 determination of cytotoxicity), a cytotoxic effect is present, in case of a $30 \%$ reduction in cell viability. ${ }^{[42]}$ Hence, the IP-S resin can be considered not toxic in these experiments even after $48 \mathrm{~h}$ of direct contact exposure. In the experiments where the growth medium was exposed to the cured resin, and later used for cell culture (Figure 8), the decrease in cell viability is again low with less than $30 \%$ in all cases. There is, however, a more significant decrease in viability compared with the experiments, where the cells were grown directly on IP-S resin (Figure 8a). The largest reduction was found for the experiments, where the extraction vehicle was kept in a thermal mixer, as shown in Figure $8 \mathrm{~b}$. This might be due to an extra step in the extraction process in the thermal mixer, as the mixer was kept running at $500 \mathrm{rpm}$, which can provide homogeneity. Another reason can be the change of $\mathrm{pH}$ in the extraction vehicle that was not kept in appropriate $\mathrm{CO}_{2}$ conditions. McCoy's $5 \mathrm{~A}$ modified medium requires $5-10 \% \mathrm{CO}_{2}$ levels to maintain physiological $\mathrm{pH}$ conditions. If not supplied, the sodium bicarbonate buffer system that this medium possesses may lose its buffering effect

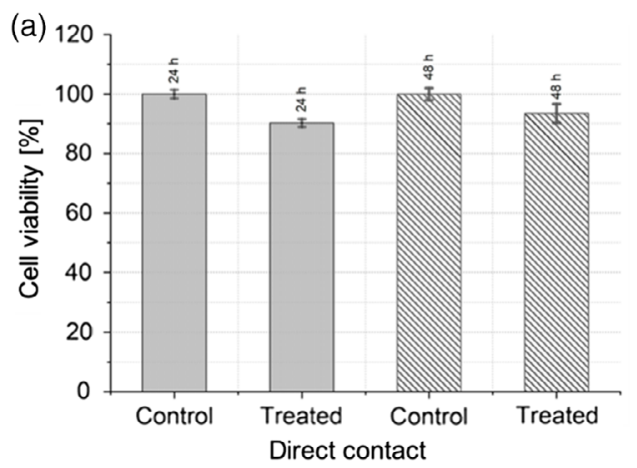

(b)

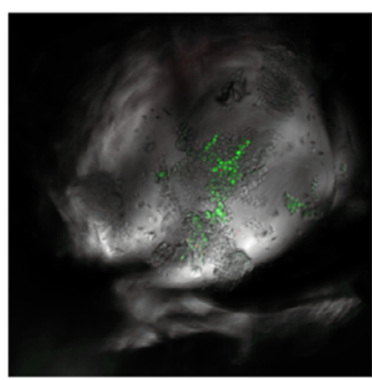

(c)

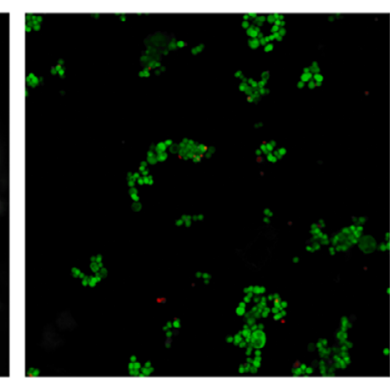

Figure 7. a) Cell viability of HCT 116 cells plated in 24 -well plates in direct contact with cured IP-S resin after 24 and $48 \mathrm{~h}$, with controls taken as $100 \%$ viability. b) Live/dead viability assay on HCT 116 cells grown on cured IP-S resin, showing the resin piece in gray, live cells in green, and dead cells in red. c) HCT 116 cells after live/dead viability assay, showing live cells stained with calcein AM and dead cells stained in ethidium homodimer-1, $48 \mathrm{~h}$ after seeding on cured IP-S resin.

(a)

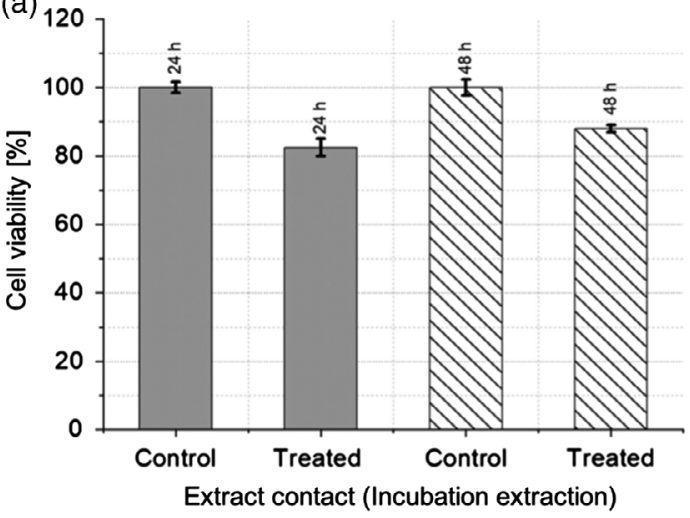

(b)

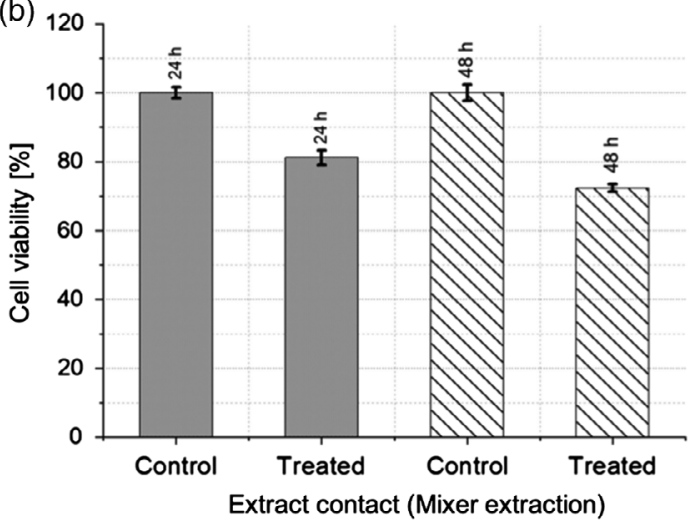

Figure 8. Cell viability of HCT 116 cells plated in 96 -well plates in contact with the extract vehicle used in a) an incubator with $95 \%$ relative humidity, $5 \% \mathrm{CO}_{2}$, at $37^{\circ} \mathrm{C}$ for $24 \mathrm{~h}$ and b) a thermal mixer at $37^{\circ} \mathrm{C}$ and $500 \mathrm{rpm}$ for $24 \mathrm{~h}$, for an IP-S cured piece after 24 and $48 \mathrm{~h}$, expressed as a percentage of control, with controls taken as $100 \%$ viability. 
and shift the medium's pH toward alkalinity values, which are not suitable for healthy cell growth. In any case, the results indicate that the cured IP-S resin has a little negative effect on cell proliferation as viability is steadily kept at more than $70 \%$ and is biocompatible.

\section{Conclusions}

The use of the high-resolution TPP 3D printing technique allowed for the robust and seamless integration of MNs with a chamber or delivery systems, for biomedical applications, circumventing the need for laborious and complex fabrication techniques. A reservoir of $2 \mathrm{~mm}^{3}$ volume topped with hollow MNs with inner diameter and height ranging from 80 to $120 \mu \mathrm{m}$ and from 200 to $400 \mu \mathrm{m}$, respectively, was fabricated. It can be further integrated with actuation and pumping mechanisms for drug delivery in future work. Investigating the outgoing flow rate through MNs using FEM and experiment for four different designs has determined that the flow profiles are laminar at an applied pressure range of $3-10 \mathrm{kPa}$. By modifying the MNs count, diameter, and shaft length, the flow rate can be modulated from 20 to $160 \mu \mathrm{L} \mathrm{s}^{-1}$. An additional analysis of the mechanical properties of the IP-S photoresist used to print the MNs has determined the elastic modulus and the yield strength of the solid resist, which were 852-1750 and 65-102 MPa, respectively. Using these mechanical properties, the buckling and fracture forces of the MNs were derived. Combined with experimental testing, this analysis verified the appropriate dimensions of the MNs that are needed to ensure mechanical stability for a given application. To corroborate the applicability of the 3D printed MNs, they were used for a penetration test into both a skin-like material and mouse skin. Penetration into skin-like material allowed the determination of the piercing force which was $0.095-0.115 \mathrm{~N}$. Confocal microscopy of the mouse skin confirmed the MN array penetration and fluorescent dye delivery 100 and $180 \mu \mathrm{m}$ deep into the skin for the 200 and $400 \mu \mathrm{m}$-long MNs, respectively. A complementary biocompatibility assessment was performed to investigate the potential of using the technique for direct tissue interfacing or implants, and it has determined that the photoresist has minimal cytotoxicity, which makes it ideal for such applications.

\section{Supporting Information}

Supporting Information is available from the Wiley Online Library or from the author.

\section{Acknowledgements}

This work was funded and supported by King Abdullah University of Science and Technology (KAUST). The authors thank Dr. Simona Spinelli, Francesco Rottoli, and StefanoPietro Mandaglio from the Animal Research Core Lab (ARCL) at KAUST for their assistance with the mouse piercing experiment.

\section{Conflict of Interest}

The authors declare no conflict of interest.

\section{Keywords}

3D printing, biocompatibilities, drug deliveries, microneedles, skin penetration

Received: November 5, 2019

Published online:

[1] R. R. S. Thakur, S. J. Fallows, H. L. McMillan, R. F. Donnelly, D. S. Jones, J. Pharm. Pharmacol. 2014, 66, 584.

[2] X. Jin, D. D. Zhu, B. Z. Chen, M. Ashfaq, X. D. Guo, Adv Drug Delivery Rev. 2018, 127, 119.

[3] A. Michaels, S. Chandrasekaran, J. Shaw, AlChE J. 1975, 21, 985.

[4] K. J. Lee, S. H. Park, J. Y. Lee, H. C. Joo, E. H. Jang, Y.-N. Youn, W. Ryu, J. Controlled Release 2014, 192, 174.

[5] M. A. Chaudhary, L.-W. Guo, X. Shi, G. Chen, S. Gong, B. Liu, K. C. Kent, J. Controlled Release 2016, 233, 174.

[6] J. Lee, D.-H. Kim, K. J. Lee, I. H. Seo, S. H. Park, E. H. Jang, Y. Park, Y.-N. Youn, W. Ryu, J. Controlled Release 2017, 268, 237.

[7] Y. Yi, J. Kosel, Sens. Actuators, A 2017, 261, 177.

[8] S. Amara, G. Sevilla, A. Torres, M. Hawsawi, Y. Mashraei, H. Mohammed, M. E. Cruz, Y. Ivanov, S. Jaiswal, G. Jakob, Adv. Eng. Mater. 2018, 20, 1800471.

[9] O. Yassine, A. Zaher, E. Q. Li, A. Alfadhel, J. E. Perez, M. Kavaldzhiev, M. F. Contreras, S. T. Thoroddsen, N. M. Khashab, J. Kosel, Sci. Rep. 2016, 6, 28539.

[10] E. Larrañeta, M. T. McCrudden, A. J. Courtenay, R. F. Donnelly, Pharm. Res. 2016, 33, 1055.

[11] M. N. Kavaldzhiev, J. E. Perez, R. Sougrat, P. Bergam, T. Ravasi, J. Kosel, Sci. Rep. 2018, 8, 9918.

[12] S. Indermun, R. Luttge, Y. E. Choonara, P. Kumar, L. C. Du Toit, G. Modi, V. Pillay, J. Controlled Release 2014, 185, 130.

[13] M. Yang, J. D. Zahn, Biomed. Microdevices 2004, 6, 177.

[14] K. Takei, T. Kawashima, T. Kawano, H. Kaneko, K. Sawada, M. Ishida, Biomed. Microdevices 2009, 11, 539.

[15] E. Mukerjee, S. Collins, R. Isseroff, R. Smith, Sens. Actuators, A 2004, 114, 267.

[16] B. Stoeber, D. Liepmann, J. Microelectromech. Syst. 2005, 14, 472.

[17] P. M. Wang, M. Cornwell, J. Hill, M. R. Prausnitz, J. Invest. Dermatol. 2006, 126, 1080.

[18] J.-H. Park, Y.-K. Yoon, S.-O. Choi, M. R. Prausnitz, M. G. Allen, IEEE Trans. Biomed. Eng. 2007, 54, 903.

[19] R. F. Donnelly, R. Majithiya, T. R. R. Singh, D. I. Morrow, M. J. Garland, Y. K. Demir, K. Migalska, E. Ryan, D. Gillen, C. J. Scott, Pharm. Res. 2011, 28, 41.

[20] S. P. Davis, W. Martanto, M. G. Allen, M. R. Prausnitz, IEEE Trans. Biomed. Eng. 2005, 52, 909.

[21] C. G. Li, C. Y. Lee, K. Lee, H. Jung, Biomed. Microdevices 2013 $15,17$.

[22] K. Kim, D. S. Park, H. M. Lu, W. Che, K. Kim, J.-B. Lee, C. H. Ahn, J. Micromechan. Microeng. 2004, 14, 597.

[23] L. M. Strambini, A. Longo, A. Diligenti, G. Barillaro, Lab Chip 2012, 12, 3370.

[24] C. G. Li, H.-A. Joung, H. Noh, M.-B. Song, M.-G. Kim, H. Jung, Lab Chip 2015, 15, 3286.

[25] S. J. Moon, S. S. Lee, H. Lee, T. Kwon, Microsyst. Technol. 2005, $11,311$.

[26] I. Xenikakis, M. Tzimtzimis, K. Tsongas, D. Andreadis, E. Demiri, D. Tzetzis, D. G. Fatouros, Eur. J. Pharm. Sci. 2019, 104976.

[27] F. Lacan, S. Coulman, A. Hotston, P. Petkov, J. Birchall, presented at Proceedings of the 10th International Conference on Multi-Material Micro Manufacture, Singapore, October 2013. 
[28] C. P. P. Pere, S. N. Economidou, G. Lall, C. Ziraud, J. S. Boateng, B. D. Alexander, D. A. Lamprou, D. Douroumis, Int. J. Pharm. 2018, 544, 425.

[29] Y. Lu, S. N. Mantha, D. C. Crowder, S. Chinchilla, K. N. Shah, Y. H. Yun, R. B. Wicker, J.-W. Choi, Biofabrication 2015, 7, 045001.

[30] A. Kundu, T. Ausaf, S. Rajaraman, Micromachines 2018, 9, 85.

[31] C. Farias, R. Lyman, C. Hemingway, H. Chau, A. Mahacek, E. Bouzos, M. Mobed-Miremadi, Bioengineering 2018, 5, 59.

[32] M. A. Luzuriaga, D. R. Berry, J. C. Reagan, R. A. Smaldone, J. J. Gassensmith, Lab Chip 2018, 18, 1223.

[33] L. Strambini, A. Longo, S. Scarano, T. Prescimone, I. Palchetti, M. Minunni, D. Giannessi, G. Barillaro, Biosens. Bioelectron. 2015, 66, 162.

[34] K. Kim, J.-B. Lee, Microsyst. Technol. 2007, 13, 231.

[35] K. Moussi, J. Kosel, J. Microelectromech. Syst. 2018, 27, 472.

[36] K. Moussi, A. Bukhamsin, J. Kosel, 20th International Conference on Solid-State Sensors, Actuators and Microsystems Q Eurosensors XXXIII (TRANSDUCERS Q EUROSENSORS XXXIII), IEEE, Piscataway, New Jersey, US, 2019.

[37] S. P. Davis, B. J. Landis, Z. H. Adams, M. G. Allen, M. R. Prausnitz, J. Biomech. 2004, 37, 1155.
[38] P. Khanna, K. Luongo, J. A. Strom, S. Bhansali, J. Micromech. Microeng. 2010, 20, 045011.

[39] Nanoscribe Photonics Professional GT, https://www.nanoscribe.de/ en/products/photonic-professional-gt/ (accessed: March 2019).

[40] X. Liang, S. A. Boppart, IEEE Trans. Biomed. Eng. 2010, 57, 953.

[41] Z. Wang, A. A. Volinsky, N. D. Gallant J. Appl. Polym. Sci. 2014, 131.

[42] International Organization for Standardization. Biological Evaluation of Medical Devices - Part 5: Tests for in Vitro Cytotoxicity, (ISO/DIS Standard No. 10993-5), 2009.

[43] International Organization for Standardization. Biological Evaluation of Medical Devices - Part 12: Sample Preparation and Reference Material, (ISO/DIS Standard No. 10993-12), 2012.

[44] N. Roxhed, T. C. Gasser, P. Griss, G. A. Holzapfel, G. Stemme, J Microelectromech. Syst. 2007, 16, 1429.

[45] W. Martanto, J. S. Moore, T. Couse, M. R. Prausnitz, J. Controlled Release 2006, 112, 357.

[46] S. D. Gittard, B. Chen, H. Xu, A. Ovsianikov, B. N. Chichkov, N. A. Monteiro-Riviere, R. J. Narayan, J. Adhes. Sci. Technol. 2013, 27, 227. 